\title{
Green computing and communications
}

\author{
Samee Ullah Khan • Lizhe Wang • \\ Laurence T. Yang • Feng Xia
}

Published online: 9 November 2011

(C) Springer Science+Business Media, LLC 2011

\section{Introduction}

Computing and communication systems play key roles in modern information technology (IT) infrastructure, affecting every aspect of life, including services, such as health, banking, commerce, defense, education, and entertainment. There are tremendously increasing demands for computing, communication, and storage systems since the last two decades. To fulfill such requirements, modern IT infrastructures have been extended and scaled, which brings various "green" related issues, such as energy consumption, heat dissemination, greenhouse emission, and resource total cost of ownership.

To make efficient use of computing and communication systems, and reduce their environmental and social impact, green computing and communication research has increasingly become a hot topic of interest for the computer research and industry.

\footnotetext{
S.U. Khan $(\bowtie)$

Department of Electrical and Computer Engineering, North Dakota State University, Fargo, ND, USA

e-mail: samee.khan@ndsu.edu

L. Wang

School of Informatics and Computing, Indiana University, Bloomington, IN, USA

e-mail: wanglizh@indiana.edu

L.T. Yang

Department of Computer Science, St. Francis Xavier University, Antigonish, NS, Canada e-mail: 1tyang@stfx.ca

F. Xia

School of Software, Dalian University of Technology, Dalian, China

e-mail: f.xia@ieee.org
} 
The study of green computing and communication attempts to alleviate this problem by delivering both high performance and reduced power consumption; thereby, effectively maximizing total system efficiency.

\section{Selected papers for the special issue}

"Review of Performance Metrics for Green Data Centers: A Taxonomy Study" is a thorough survey and classification of the performance metrics and indicators for green (or energy-efficient) data centers.

"A Localization Method for the Internet of Things" addresses efficient and effective information acquisition and localization for Internet of Things paradigm. The theory is verified through the setup of a trial system that certifies the feasibility of the approach.

"Massively Parallel Modeling and Simulation of Large Crowd with GPGPU" provides insights on simulating crowds and crowd behaviors on a GPGPU.

"Designing Energy Efficient Communication Runtime Systems: A View from PGAS Models" details the development of a communications library that exploits the slack in communications to save energy consumption through dynamic voltage and frequency scaling.

"Performance Modeling and Optimization of Sparse Matrix-Vector Multiplication on NVIDIA CUDA Platform" reports the various characteristics and performance results pertaining to Sparse Matrix-Vector Multiplication, such as cache misses and frequency of accessing data.

"A Workload-Driven Approach to Database Query Processing in the Cloud" addresses data provisioning issues with the cloud computing paradigm by considering workload characters and patterns.

Acknowledgements The guest editors are in debt to the knowledgeable reviewers that delivered timely assessments on the research articles submitted to this special issue-thank you. 